\title{
Les immigrants au Japon passés au crible commun du jus fusionis
}

Perspective historique

Foreign immigrants in Japan and jus fusionis. A historical perspective

\section{Marc Humbert}

\section{(2) OpenEdition}

\section{Journals}

Édition électronique

URL: http://journals.openedition.org/transcontinentales/789

DOI : 10.4000/transcontinentales.789

ISBN : 978-2-7351-1557-0

ISSN : 1775-397X

\section{Éditeur}

Editions de la maison des sciences de l'homme

\section{Édition imprimée}

Date de publication : 31 décembre 2010

ISSN : 1950-1684

\section{Référence électronique}

Marc Humbert, "Les immigrants au Japon passés au crible commun du jus fusionis »,

Transcontinentales [En ligne], 8/9 | 2010, document 4, mis en ligne le 31 décembre 2010, consulté le 08 septembre 2020. URL : http://journals.openedition.org/transcontinentales/789 ; DOI : https://doi.org/ 10.4000/transcontinentales.789

Ce document a été généré automatiquement le 8 septembre 2020.

Tous droits réservés 


\title{
Les immigrants au Japon passés au crible commun du jus fusionis
}

\author{
Perspective historique \\ Foreign immigrants in Japan and jus fusionis. A historical perspective
}

\section{Marc Humbert}

1 Partout les migrants rêvent d'un séjour agréable dans un pays où ils pourront travailler et vivre comme ceux qui, depuis des générations, s'y trouvent bien; certains vont même jusqu'à imaginer y faire souche. Au regard de ce rêve, le Japon se présente comme l'un des moins accueillants de tous les pays industriels.

2 Les immigrants, c'est-à-dire les étrangers présents sur le territoire japonais pour un séjour dépassant les trois mois autorisés sans visa pour le " tourisme ${ }^{1}$ ", formaient, en $2005^{2}$, seulement $1,6 \%$ d'une population totale de près de 130 millions de Japonais. Ce pourcentage est à comparer avec celui que l'on trouve en France $(10,6 \%)$, aux ÉtatsUnis (13\%), au Royaume Uni (9,7 \%), ou encore en Allemagne (12,9\%).

3 Le Japon est également dix fois plus fermé que la France à l'accueil des réfugiés. En $2007^{3}$, ceux-ci n'étaient que 1800 au Japon et 1500 d'entre eux attendaient que l'on statue sur leur sort, tandis qu'ils étaient au nombre de 152000 en France (avec 31000 en attente) ou de 300000 en Allemagne (avec 11000 en attente). Le Japon n'a signé qu'en 1981, sous une forte pression internationale, la convention de 1951 sur la protection des réfugiés ratifiée en 1954 par la France.

4 En outre, ces étrangers vivant au Japon ne sont pas bien accueillis. Le centre de recherches du mensuel économique britannique The Economist a calculé un indice d'accessibilité concernant les conditions d'embauche, les facilités de réunification familiale, les programmes d'intégration ${ }^{4}$, etc. ; sur ce critère, le Japon est loin derrière tous les pays industrialisés, et même, derrière la Corée, l'Inde et la Chine ${ }^{5}$.

5 Pour compléter le tableau de cette forte réticence japonaise vis-à-vis des étrangers, il faut ajouter que le pourcentage d'immigrés obtenant la nationalité japonaise reste lui aussi très faible. Depuis une dizaine d'années, il est de l'ordre de 15000 naturalisations par an. Ce chiffre est aussi dix fois moins grand que celui de la France qui a naturalisé, 


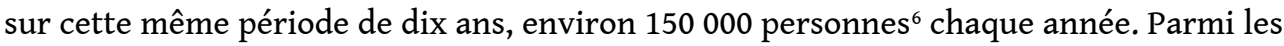
naturalisations japonaises des dix dernières années, plus de $95 \%$ concernaient des personnes d'origine coréenne ou chinoise.

6 Après avoir présenté les données de la discrimination administrative et grégaire entre les Japonais et les étrangers, j'apporterai des éléments de réflexion sur ce qui fonde cette discrimination : l'application souvent implicite à tout individu au Japon du crible commun d'un jus fusionis.

\section{La discrimination administrative et grégaire}

7 Les données administratives utilisent pour les étrangers séjournant plus de trois mois au Japon plusieurs catégories de statuts dont les dénominations ne font pas bien apparaître leurs différences : résident permanent au titre du traité, résident permanent général, résident permanent spécial, résident de long terme, stagiaires, activités désignées, conjoint ou enfants de Japonais ou de résident permanent. Elles ont été créées en lien avec des questions concernant trois ou quatre pays d'origine qui, jusqu'ici, ont joué successivement un rôle essentiel dans l'immigration au Japon: la Corée, le Brésil, la Chine et, dans une moindre mesure, les Philippines. Je vais décrire à grands traits les étapes de la mise en place de la discrimination administrative par des lois de contrôle de l'immigration puis présenter une grille de lecture du comportement grégaire des Japonais vis-à-vis des non-Japonais.

\section{Les étapes de la mise en place des outils de la discrimination administrative}

1952-1992

8 La Corée ayant été annexée par le Japon en 1910, les Coréens - comme les ressortissants d'autres colonies nipponnes - étaient devenus administrativement des Japonais. Sur les deux millions qui avaient été amenés à vivre au Japon, un grand nombre sont retournés chez eux après 1945. Toutefois, en 1952, quand le Japon a recouvré sa souveraineté et inauguré sa toute nouvelle démocratie, un demi-million de Coréens avaient choisi de rester et de faire du Japon leur pays. Ils ont alors perdu, du jour au lendemain ${ }^{7}$, leur nationalité japonaise (comme un certain nombre de ressortissants d'autres colonies nipponnes, les Taïwanais et aussi les Chinois de Mandchourie, par exemple). Depuis, ils ont été traités comme des étrangers et dépourvus des droits économiques, culturels et sociaux associés au statut de Japonais. Ils sont pourtant demeurés au Japon ${ }^{8}$ et ont rechigné à demander la nationalité japonaise qu'ils considéraient avoir injustement perdue. En 1965, ils n'étaient que 30000 à l'avoir à nouveau obtenue ${ }^{9}$. À cette date, leur situation d'étranger était administrativement clarifiée, ils avaient en effet bénéficié du statut de résident permanent au titre du traité (kyotei-eiju), à la suite de l'accord de normalisation signé entre le Japon et la Corée du Sud ${ }^{10}$.

Pendant trente ans, les Coréens ont été quasiment les seuls étrangers au Japon: jusqu'en 1980, ils y constituaient plus de $85 \%$ de la population étrangère. Leur nombre total a peu évolué mais a continué de croître jusqu'en 1992, y compris avec de nouveaux entrants ne bénéficiant pas de l'accord de $1965^{11}$. Il était prévu que cet accord soit révisé en 1991, vingt-cinq ans après, pour envisager la situation des Coréens de la 
génération suivante. Mais, avant cela, dès la ratification en 1981 de la convention sur les réfugiés (voir supra), des dispositions ont été appliquées pour le bénéfice des Coréens. En outre, la nouvelle loi de 1982 sur le contrôle de l'immigration a permis aux Coréens (Zainichi,résidents au Japon) qui n'avaient pas opté pour la Corée du Sud et ne bénéficiant pas de l'accord de 1965 d'obtenir le statut de résident permanent général (ippan-eiju). Quelques autres améliorations virent le jour également pour les enfants.

10 À l'occasion des discussions pour le renouvellement de l'accord de 1965, l'administration japonaise a pris en considération les descendants de Japonais (nikkei) qui avaient émigré en Asie et dont un certain nombre revenaient au Japon avec leur famille. Le renouvellement donne le memorandum de 1991 par lequel tous les Coréens obtiennent de manière automatique le statut de résident permanent spécial (tokubetsueiju) et bénéficient de quelques droits sans bien sûr obtenir tous ceux des citoyens japonais.

11 Le nombre des Coréens, parfois dénommés « anciens venus » (oldcomers, ôrudo kamâ), a décliné après $1992^{12}$, surtout en termes relatifs dans une population étrangère qui s'accroissait, atteignant puis dépassant le million de personnes en 1992, pour parvenir à plus de deux millions en 2007.

12 Le relais a été pris par de nouveaux venus (newcomers, nyûkamâ ou shinrai), tout d'abord des Chinois puis, à un moindre degré, des Brésiliens et des Philippins. En 1990, il y avait déjà au Japon plus de 100000 Chinois pour 42000 Brésiliens et 36000 Philippins. À partir de 2000, le chiffre de la population chinoise décolle et elle devient, en 2007, la première population étrangère au Japon devant les Coréens.

\section{Le tournant 1985-1994}

13 Jusque 1985, le patronat japonais n'a pas cherché à faire venir de la main-d'œuvre étrangère, préférant automatiser intensément le processus de production tandis que la politique économique et celle du change permettaient le plein emploi et l'élévation des salaires. À partir de 1985 et des accords du Plaza, la croissance japonaise manque de main-d'œuvre. Les entreprises découvrent à ce moment les Brésiliens descendants de Japonais, des nikkei ${ }^{13}$, alors que le Brésil est en crise et que les Brésiliens cherchent du travail à l'étranger. Pendant que des entreprises privées mettent en place un réseau de recrutement de main-d'œuvre, l'administration japonaise inventent pour les nikkei de Chine et d'Asie (voir supra) un dispositif qui va servir surtout pour les nikkei sudaméricains, principalement les Brésiliens.

Jusque 1990, des Brésiliens fils de Japonais - en raison de l'application du jus sanguinis pouvaient venir travailler avec un visa de fils ou fille de citoyens japonais (issei, première génération). Venus pour travailler hors de chez eux, ils ont été nommés dekasegi. L'application aux Brésiliens de la nouvelle loi du contrôle de l'immigration votée en 1989 et créant le statut de résident de long terme (teijûsha) a permis à des descendants de Japonais de deuxième et troisième générations (nissei et sansei) de venir travailler au Japon. Ils ont été de plus en plus nombreux à s'installer avec leur famille pour y faire naître et éduquer leurs enfants, sans avoir pour autant une citoyenneté japonaise à part entière ${ }^{14}$. Cette population étrangère s'est accrue rapidement, elle a atteint 200000 personnes en 2000 avec de fortes concentrations régionales. Son implantation dans les communautés locales a posé des problèmes que le Japon n'avait pas connus avec les « immigrants » coréens qui vivaient au Japon depuis un demi-siècle 
ou plus. Les Brésiliens forment la troisième population étrangère derrière les Coréens et les Chinois. Ces derniers ont été par ailleurs la première population bénéficiaire d'une nouvelle législation sur les stagiaires présentée ci-après.

Les agences de recrutement japonaises, en l'absence d'une législation du travail adaptée, ne pouvaient placer des travailleurs temporaires dans l'industrie manufacturière, jusqu'à la loi de $2004^{15}$. La législation antérieure fut contournée grâce à une formule-type pour les stages, étendue aux migrants par la loi du programme de stagiaires techniques, en 1993. Certains étrangers passèrent du statut de stagiaire à celui d'activités désignées. Ces deux statuts limitent étroitement les conditions et les métiers dans lesquels un travail peut être exercé, pour une durée de trois ans maximum sans possibilité de changer d'emploi. Les autres statuts, y compris celui de conjoint ou d'enfant de Japonais, n'impliquent pas les mêmes restrictions en matière de travail.

Le mariage avec un Japonais est une autre source d'augmentation de la population étrangère, en particulier chinoise, au Japon. Il ne confère pas la nationalité japonaise mais permet d'obtenir ce statut de conjoint ou d'enfant de Japonais et la possibilité d'occuper un emploi dans de bonnes conditions. Environ $6 \%$ des mariages au Japon sont des mariages avec un conjoint non japonais. Dans $80 \%$ des unions, un Japonais convole avec une étrangère qui est, dans $38 \%$ des cas, une Chinoise. Nombre de mariages sont arrangés par des agences pour des agriculteurs japonais âgés.

\section{L'avenir après 2010}

La crise de 2009, surtout celle de l'industrie, a touché de plein fouet les Brésiliens employés comme travailleurs temporaires dont les contrats n'ont pas été renouvelés. Le Japon leur a offert une aide au retour ${ }^{16}$ sous réserve de ne pas revenir avant trois ans sous le même statut. En 2009, le nombre total d'étrangers au Japon a diminué pour la première fois depuis 1961. Les données officielles indiquent qu'ils forment 1,71 \% de la population totale. Le nombre de Brésiliens a diminué de 45000 , le nombre de Coréens de plus de 10000 , seule la population étrangère Chinoise a continué de s'accroître fortement : plus de 25000 personnes. Les Chinois représentent désormais plus de $30 \%$ des étrangers au Japon ${ }^{17}$.

Le gouvernement japonais réfléchit à une réforme et envisage divers programmes dont certains sont déjà en place pour faire venir des travailleurs asiatiques dans un certain nombre de professions pour des flux qui restent dérisoires. On est loin des rares appels à une politique active d'immigration pour compenser le vieillissement et le déclin de la population. En outre, une réforme politique et administrative pourrait buter sur la perpétuation d'un type de comportement tel, que la population japonaise tend à se souder et à ne pas accepter facilement ceux qui ne s'inscrivent pas dans le moule commun ${ }^{18}$.

\section{La lecture du comportement grégaire vis-à-vis des non-Japonais}

19 Fukuoka (2000) a élaboré une grille de lecture de cette frontière invisible qui, au sein de la population permanente de l'Archipel, sépare Japonais et non-Japonais et rend la vie difficile à ces derniers. Selon lui, trois caractéristiques jouent un rôle essentiel :

20 a) être japonais par le sang, par la descendance, par une filiation avec des personnes considérées ethniquement comme japonaises ; 

ne pas avoir la nationalité japonaise. C'est le cas des issei,enfantsd'émigrés japonais qui, venant au Japon, obtiennent le statut de conjoint ou enfant de Japonais. Rentrent aussi dans cette catégorie, des Japonaises mariées à un Coréen avant 1952 et qui ont perdu leur nationalité japonaise à cette date.

Tableau 1

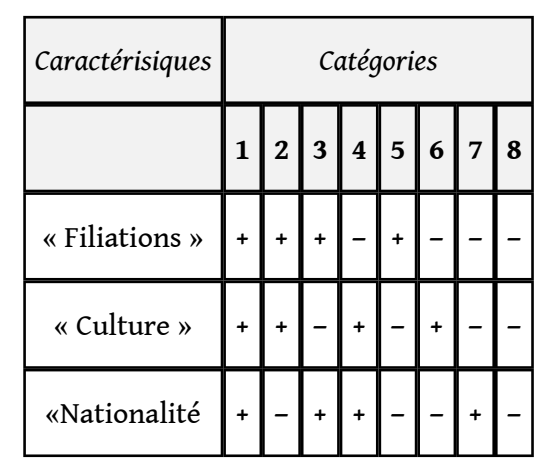

Les catégories de Japonais et de non-Japonais

Source: Fukuoka (2000)

Dans la catégorie [3], on trouve des personnes japonaises par le sang, ayant la nationalité japonaise, mais qui ne parlent pas japonais. Certaines ont perdu ou n'ont jamais eu la pratique de la langue ou/et d'autres éléments de la culture japonaise. C'est en particulier le cas d'enfants ayant vécu ou même qui sont nés à l'étranger de Japonais expatriés. Ayant internalisé une culture étrangère, ces enfants retournés au pays (kikoku shijo) rencontrent de nombreuses difficultés d'adaptation, notamment à l'école, même vis-à-vis de leurs camarades d'école. On organise parfois pour eux des cours de rééducation puisqu'ils sont considérés comme n'étant plus pleinement japonais.

Dans la catégorie [4], nous avons des personnes ayant la nationalité japonaise, qui parlent japonais et se comportent selon la culture japonaise, mais qui ne sont pas japonaises par le sang. C'est le cas par exemple de Coréens d'origine qui ont été élevés au Japon et qui ont pris la nationalité japonaise. Ils se sentent pleinement japonais, mais la société japonaise ne les considère pas totalement comme tels, en particulier 
lorsqu'ils veulent se marier et qu'il leur faut présenter leur registre d'état-civil. Un promis ou une promise peut alors rencontrer l'opposition des parents du futur conjoint qui est pleinement japonais.

La catégorie [5] est formée des personnes d'ascendance japonaise mais qui ont perdu l'usage de la langue et des comportements culturels japonais ainsi que la nationalité japonaise. C'est le cas des descendants de Japonais émigrés au Brésil. L'opinion publique a eu d'abord le sentiment que ces personnes étaient fondamentalement japonaises. Mais, lors de leur installation, bien que la plupart aient un physique japonais, elles ont eu un comportement social qui perturbait trop les habitudes. Comme en outre la quasitotalité d'entre elles ne parlaient ni n'écrivaient le japonais, une partie importante de la population, en dépit des premiers sentiments de sympathie et de nostalgie, est devenue plus que réticente à leur égard. On trouve aussi dans cette catégorie les orphelins de la guerre descendants de Japonais, élevés en Mandchourie ou ailleurs (Chugoku zanryu koji), qui rencontrent la même réticence.

Pour la catégorie [6], il faut des personnes parlant et écrivant le japonais, se comportent selon les habitudes culturelles japonaises, mais qui ne sont pas d'ascendance japonaise et qui n'ont pas la nationalité japonaise. Ce sont pour tous les pays d'excellents candidats à la naturalisation mais on a vu que le Japon est avare de sa nationalité pour les immigrants. De fait, beaucoup de Coréens restés après 1952 et leurs descendants nés au Japon sont dans cette situation. Ils rencontrent un peu plus de problèmes que leurs congénères de la catégorie [4].

31 Le Japon étant réticent à octroyer sa nationalité, il n'est normalement pas possible d'avoir, dans l'Archipel, des populations de la catégorie [7], c'est-à-dire qui ont la nationalité japonaise mais ont une filiation et des pratiques culturelles différentes. C'est cependant le cas d'une partie des personnes vivant dans les territoires - annexés tardivement - des îles du sud et du nord de l'Archipel ; appartenant au clan de Satsuma pour les Ryukyu (Okinawa) ou au clan de Matsumae pour les Aïnu, ces populations non japonaises payaient tribut ${ }^{19}$.

32 La plupart ont été fortement « assimilées » et les Aïnu sont peu nombreux; en revanche la population d'Okinawa, nombreuse, maintient une certaine différence visible. Ses comportements culturels ont des spécificités marquées et la langue okinawaienne, certes proche du japonais mais différente, reste vivante.

Toutefois, s'il existe un sentiment que les Okinawaiens ne sont pas pleinement japonais, cela ne semble pas poser à cette population des problèmes comparables à ceux des autres catégories: ils bénéficient à titre individuel des mêmes droits que tous les citoyens japonais et ne sont pas victimes de pratiques sociales discriminatoires ce qui a été, en revanche, souvent le cas pour des descendants d'Aïnucherchant à pouvoir afficher leur différence.

\section{La fabrication historique d'un crible commun pour être japonais : le jus fusionis}

34 Je vais présenter à grands traits le contexte historico-politique de la mise en place d'un discours culturaliste sans pouvoir en peu de mots étayer parfaitement ma présentation. Il faut lire sur ce point des travaux essentiels de Berque et d'autres que je cite. Cette 
construction idéologique, qui ne dit pas son nom, élabore une sorte de jus fusionis qui, à certains égards, mène la société japonaise dans une impasse.

\section{Le contexte historique d'un discours sur la manière d'être Japonais}

35 Jusqu'à la restauration Meiji ${ }^{20}$, l'empereur ne jouait pas un rôle exorbitant aux yeux des Japonais pour maintenir l'intégrité du pays, formulation qui sera plus tard remplacée par «maintenir l'unité nationale». Le Japon était une oligarchie aristocratique guerrière, subdivisée en fiefs, qui dominait une population constituée principalement de paysans, cyniquement comparés à des graines de sésame qui donnent d'autant plus d'huile qu'on les écrase plus fortement. Il y avait certes plus pauvres que les plus pauvres: les différentes catégories de non-humains, et les populations non-japonaises qui payaient tribut (voir supra). Au centre, les populations japonaises vivaient leurs diversités et parlaient différents dialectes sans aucune injonction du pouvoir central. Le bouddhisme était la religion dominante pour raison d'État, mais avec de nombreuses sectes, et les monastères organisaient parfois des écoles (terakoya) pour le peuple. La religion Shintô ne tenait pas, loin s'en faut, une place importante.

La situation a fortement changé à l'ère Meiji ${ }^{21}$. La réorganisation du pouvoir autour de l'empereur qui doit prendre une importance première rend aussi nécessaire la restauration de la religion Shintô dont il est le personnage principal. Il faut également, pour sauvegarder l'indépendance, se mettre semble-t-il du côté des envahisseurs. Certes, le slogan est celui de chasser les barbares, mais c'est à l'extérieur que le Japon va chasser. Dès le début de l'ère Meiji, la volonté d'aller conquérir la Corée est présente, et le début du siècle montre un Japon agressif qui a déjà annexé les Ryukyu en 1879, colonisé Taïwan en 1895, et va prendre possession de la Corée en 1910. Il ne s'agit plus de se fermer vis-à-vis de l'étranger comme à l'époque d'Edo (1600-1868) mais d'aller agresser l'étranger. Pour mener à bien de telles guerres extérieures, il faut des soldats et souder derrière eux les masses populaires, leur expliquer la pression très forte et menaçante de l'Occident, pression militaire, économique et intellectuelle.

En quelques décennies, la réorganisation bureaucratique va trouver le soutien d'intellectuels japonais qui vont produire un discours sur l'essence culturelle propre du Japon et en construire la théorie sur une logique de type occidental. Ce discours intellectuel va permettre de justifier le discours et les pratiques politiques de la bureaucratie gouvernementale autocrate et militariste. Lors de l'ère Taisho cependant, une certaine démocratie s'installe pendant un moment mais elle ne durera pas. On assiste ensuite à la montée d'un État totalitaire et militarisé qui s'allie aux États fascistes européens et mène des guerres d'agression à l'extérieur, terrorisant et endoctrinant le peuple à l'intérieur. L'issue sera la défaite de 1945, mais une grande part de l'élite politique et intellectuelle et un certain nombre d'institutions retrouveront leur place après sept années sous l'occupation et l'administration des États-Unis. Des intellectuels japonais reprendront la plume pour peaufiner le même discours, relayé à l'occasion par des hommes politiques

"Le discours sur une homogénéité ethnique japonaise maintenue depuis les temps anciens n'émane pas seulement de conservateurs nostalgiques du kokutai [théorieidéologie du début $\mathrm{du} \mathrm{xx}^{\mathrm{e}}$ siècle selon laquelle le pays fait corps, avec en son centre, l'empereur] mais également d'historiens "de gauche" comme Tôma Seita ou Fujitani Toshio $^{22}$ ». Citons Masuda Yoshio : «Les Japonais, comme groupe ethnique homogène, 
ont gardé une culture homogène depuis les temps préhistoriques ${ }^{23}$.» Ce discours n'est pas seulement un discours d'intellectuels, il est relayé dans les medias et par des hommes politiques importants. Ainsi le Premier ministre Nakasone (1982-1987) affirmait $^{24}$, en 1986, que "les Japonais constituent une ethnie unique (tan'itsu minzoku) ». Tandis qu'en 2005, le ministre des Affaires étrangères de Koizumi, Taro Aso, déclarait au journal Yomiuri (Burgess 2005) que le Japon est "une nation, une civilisation, une langue, une culture et une race ». La grille de Fukuoka utilisée dans la section précédente correspond donc bien à une réalité perçue et dite comme telle dans la société japonaise.

\section{L'instauration de la « nipponitudologie » (nihonjin ron) et le « nipponitudologisme »}

39 Au début du xx siècle, quelques intellectuels cherchent à construire une théorie afin de définir et de revendiquer une essence « culturelle » propre au Japon et aux Japonais. La démarche de nombre d'entre eux fait abstraction de toute implication politique, mais pour d'autres, et plus encore pour des bureaucrates et des politiciens, l'ambition est de mettre cette théorie au service de la construction d'un pouvoir, d'un État fort.

Ce courant intellectuel va construire ce qui sera dénommé après la deuxième guerre mondiale nihonjin ron, un discours sur les Japonais, en fait, plus exactement, une théorie sur la manière d'être Japonais. Pour être explicite, il faut dire en français la théorie de la nipponité ou de la nipponitude - la Nippon Attitude -, soit la " nipponitudologie ». Cette théorie n'est pas une conceptualisation de la manière d'être des Japonais telle que ce courant intellectuel peut l'observer ou la reconstruire en étudiant l'histoire des décennies ou du siècle précédent. L'objectif est d'exprimer l'essence profonde, supposée, la version idéale, de la manière d'être Japonais.

41 Ses premiers contributeurs se réfèrent en cette matière plus large à la démarche qui avait été celle de Motoori Norinaga (1730-1801) pour définir l'essence de la littérature en japonais par rapport à la littérature en chinois, à partir de mono no aware, compris comme le pathos des choses, leur sensibilité. Cet auteur était un leader de la constitution d'une science nationale (kokugaku) glorifiant l'éminence du Japon " pays suprême » " parce que la sainte lignée impériale est immuable, que le riz, qui soutient la vie humaine est beau [...] le Pays Suprême est le seul à n'avoir jamais été envahi ${ }^{25}$ ».

Avec l'ère Meiji, l'entreprise intellectuelle et politique est menée non plus en opposition à la pensée chinoise et à la Chine, mais par opposition à la pensée occidentale et pour rattraper l'Occident. Mais, la manière dont est conduite l'opération paraît relever de la véritable fraude intellectuelle aux yeux de Basil Hall Chamberlain, qui est un des pionniers occidentaux des études japonaises. Il observe d'un œil critique la naissance de ce discours et la prise de mesures politiques par la bureaucratie au pouvoir qui en découle. Dans une conférence à Londres, il affirme que c'est une fraude intellectuelle qui comprend une nouvelle religion dont le culte impérial, Shintô et Bushidô sont la préfiguration. On dépoussière le Shintô discrédité pour le revivifier, on organise un prosélytisme révérencieux à l'égard de l'empereur auparavant traité de manière "cavalière ", on glorifie le code des samouraïs comme un système ancien au cœur de la culture japonaise alors qu'aucun chercheur n'avait entendu le terme de bushidô avant le tournant du $\mathrm{xx}^{\mathrm{e}}$ siècle. 

$\mathrm{du}$ sujet et de l'objet, ou encore indifférenciation entre individu et objet. Nishida souligne alors, en considérant la pensée occidentale, l'artificialité du moi et s'oppose à la «fonction discriminante de la conscience logique comme dé-authentifiant l'harmonie immédiate de l'homme avec le monde ${ }^{27}$ ». Kawakami (1911) découvre le caractère unique de son pays où «il y a une identité absolue entre l'intérêt privé individuel et l'intérêt public impérial », ce qui l'amène à affirmer l'indivisibilité de l'individu, du pays et de l'empereur. L'essence de la nipponitude résiderait dans cette non-aliénation du sujet, dans la fusion du moi et de l'autre. La pensée occidentale, en opposant sujet et objet, détruit l'harmonie qui est préservée au Japon entre les Japonais et leur monde. L'occidentalisation contraindrait donc les Japonais « à entrer et habiter dans un monde "inauthentique" de réflexion critique qui suppose une opposition dialectique entre moi et l'autre ${ }^{28}$ ». privé et de l'intérêt public, de l'importance relative de l'individu et du groupe et de la société au Japon ou en général, ne peut que susciter l'attention. En outre, en raison de l'insatisfaction en Occident également d'un grand nombre de penseurs et des 
populations face à l'individualisme exacerbé, les discours qui redonnent une place cruciale à l'insertion de l'individu dans la société sont écoutés. C'est de cette manière que s'est positionné en particulier Hamaguchi (1977) qui défend "une nouvelle conceptualisation orientale de la personnalité " dans laquelle ce qu'il appelle le kanjinshugi (entre-les-hommes-isme $=$ relationisme) est en opposition radicale avec le kojinshugi (individualisme) occidental ${ }^{29}$. Mais, dans la réalité concrète les Occidentaux, et les humains en général, ne fonctionnent pas comme des individualistes de la théorie et ont un sens de l'intérêt public. Certains sont conscients que l'intérêt public ne concorde pas toujours avec leur intérêt privé et que le raisonnement logique, le débat avec les autres, amène à la décision individuelle responsable de contribuer à l'intérêt public.

Le discours de la nipponitudologie souligne par ailleurs que, pour l'existence de l'individu lui-même, les relations avec les autres sont essentielles. C'est ce que soutient, entre autres, Heidegger auquel se réfère par exemple Watsuji. Mais chez Heidegger, la singularité de l'individu est préservée ; il faut être dans le monde (in-der-Welt-sein) et pas seulement avec (Mitsein). Watsuji comprend le monde comme la société et considère que les hommes ne sont des hommes que s'ils constituent une existence commune dans laquelle ils se fondent. Par la suite, il a écrit: "l'homme comme un individu abstrait n'existe pas et l'existence humaine universelle peut seulement être réalisée à travers la forme particulière de la nation ${ }^{30} »$. Dans ces conditions, pour être Japonais, il faut «se fondre " dans la nation japonaise. Cette exigence exprime, en quelque sorte, que la nationalité japonaise dépend du droit de la fusion dans la Nation, le jus fusionis : est-ce que cette personne fusionne ou non avec la nation japonaise?

\section{L'impasse du nipponitudologisme pour les Japonais et pour la société japonaise}

50 Le discours nihonjin ron, la nipponitudologie prend, on le voit, une posture doctrinale qui en fait une idéologie, le nipponitudologisme. Son caractère totalitaire dans ses implications politiques est clair. Ses conséquences principales ne portent pas seulement sur les relations du Japon vis-à-vis de l'étranger, mais aussi sur les relations des Japonais vis-à-vis du Japon et sur le fonctionnement de la société japonaise. Cela mérite attention pour notre propos puisque cela est directement lié à la manière dont les Coréens, par exemple ${ }^{31}$, sont traités au Japon.

51 En raison de « cette exclusion idéologique au sein de la culture japonaise d'un principe d'équilibre entre l'individu et sa société, ni la potentialité de l'individu, ni celles de la communauté ne peuvent jamais parvenir, ou aspirer à une complète réalisation ${ }^{32}$ ». Ogino (2004) illustre cette conséquence par le cas de Nagai Kafû qui a su résister au régime fasciste mais n'a pu protester, en 1911, lors du Taigyaku jiken (incident de haute trahison), l'affaire dite du crime de lèse-majesté qui a été utilisée par le pouvoir pour exécuter onze intellectuels ${ }^{33}$. Kafû s'est exprimé à ce propos : «Pour avoir crié justice lors de l'affaire Dreyfus, le romancier Zola ne s'était il pas réfugié hors de sa patrie? Pourtant, ni moi ni les autres hommes de lettres de l'époque, n'avons rien dit. [...] Dorénavant, pensai-je, il ne me restait pas d'autre voie sinon que d'abaisser la qualité de mon art au niveau de celui des divertisseurs d'Edo ${ }^{34}$.» Kafû faisait allusion à l'autocensure pratiquée par les maîtres de l'estampe japonaise. « En se renfermant dans l'autocensure, Kafû fait la démonstration de la logique de l'exclusion de l'autre dans sa forme la plus extrême, car l'autocensure n'est rien d'autre qu'une forme de l'exclusion 
de l'autre en soi. En rendant impensable la critique, ne serait-ce que sur le plan formel, Kafû balaie la possibilité de la lutte contre l'injustice telle qu'il l'a lui-même admirée chez Emile Zola ${ }^{35}$. »

La pression policière de l'État est censée avoir disparu après la défaite avec la mise en place d'une nouvelle démocratie. Ce moment est certainement, après celui de la restauration Meiji, celui d'une deuxième «révolution » de l'époque moderne pour le peuple du Japon, c'est-à-dire un profond bouleversement de sa société. Cet avis est partagé par beaucoup comme Pelletier (2007b : 46) qui écrit : « Les quelques années qui suivent la défaite, habituellement appelées "après-guerre" sont cruciales car c'est le Japon contemporain qui est redessiné, autant dans son image que dans ses structures. Elles le sont d'autant plus que les décisions prises par les dirigeants japonais et l'occupant étatsunien touchent des éléments aussi essentiels que le système impérial et le caractère pacifiste de la constitution. » Je partage volontiers cette idée que des transformations fondamentales ont eu lieu, toutefois, on a déjà montré que le nihonjin ron et le nipponitudologisme qu'il soutient, seront renforcés et dits comme tels, dans les années 1970. C'est donc que sur ce point l'aggiornamento n'a pas encore été fait. L'élément mis en avant et pour partie porteur de changement, à savoir la Constitution de 1947, porte d'ailleurs en lui-même, avec les lois et décrets que la Constitution permet de promulguer après le retour de souveraineté aux mains d'un gouvernement japonais en 1952, le maintien d'un nipponitudologisme au moins passif.

"Ce texte juridiquement fondamental, bien que décrit comme "pacifiste", garde le silence total sur les Japonais d'origine coréenne ou chinoise qui, à la veille de la mise en place de la Constitution, ont été exclus du cadre juridique japonais ${ }^{36}$ par décret unilatéral et sans concertation. »

En dépit du bel esprit de cette constitution pour la paix et à l'égard des droits de l'homme, le jus fusionis a été appliqué par une simple circulaire, décidé arbitrairement par le pouvoir tout-puissant. Et ce crible continue d'être le crible commun pour tous les résidents de l'Archipel.

\section{BIBLIOGRAPHIE}

BERQUE, A. 1977. « Problèmes et politiques migratoires au Japon », Revue Tiers Monde,t. XVIII, n 69, janvier-mars : 77-99.

BERQUE, A. 1982. Le sens de l'espace au Japon. Paris, Presses universitaires de France. L'ouvrage, après avoir été épuisé, a été repris avec Maurice Sauzet, et publié par les éditions Arguments (Paris) en 2004.

BERQUE, A. 2000. Ecoumène-Introduction à l'étude des milieux humains. Paris, Belin.

BURGESS, C. 2005. « Multicultural Japan? Discourse and the "myth" of homogeneity », The Asia-Pacific Journal : Japan Focus, http://www.japanfocus.org/products/details/2389. 
DALE, P. 1986. The Myth of Japanese Uniqueness, London, Croom Helm. Quotedfrom a fourth edition (1995), London, Routledge.

DoI, T. 1971. Amae no kôzô (Le jeu de l'indulgence). Tokyo, Kôbundô.

FUKUOKA, Y. 2000. Lives of Young Koreans in Japan. Melbourne, Trans Pacific Press.

HAMAGUCHI, E. 1977. “Nihonrashisa”no saihakken (A rediscovery of. Japaneseness.Tokyo, Nihon Keizai Shinbunsha.

HISAMATSU, S. 1963., The Vocabulary of Japanese Literary Aesthetics,trad. H. McCullough. Tokyo, Center for East Asian Cultural Studies.

KAWAKAMI, H. 1911. “Nihon dokutoku no kokkashugi,in KaWAKAMI H. Chosokushû, 8 Tokyo, Chikuma Shobô.

NAKANE, C. 1967. Tate shakai no ningen kankei.Tokyo, Kôdansha Gendai Shinsho.

NAKANE, C. 1974. La Société japonaise. Paris, Armand Colin.

NiSHIDA, K. 1910. Zen no kenkyû. Tokyo, Inawami Bunko (reprint 1979).

NOZAKI Y., H. INOKUCHI et K. TAE-YOUNG. 2006. « Legal Categories, Demographic Change and Japan's Korean Residents in the Long Twentieth Century », The Asia-Pacific journal : Japan focus, http:// www.japanfocus.org/-Yoshiko-Nozaki/2220, consulté le 7 novembre 2010.

OGINO, F. 2004. « Une culture sur fond de fermeture », in J.-F. SABOURET (dir.), Japon, peuple et civilisation. Paris, La Découverte : 40-44.

Pelletier, P. 2007a. « Le nationalisme insulaire. Théories et avatars de l'identité japonaise », in J.M. Bouissou (dir.), Le Japon contemporain. Paris, Fayard : 369- 391.

Pelletier, P. 2007b. Le Japon. Géographie, géopolitique et géohistoire.Paris, Sedes Armand Colin.

SEKIGUCHI, T. (2002) «Nikkei Brazilians in Japan : Hopes and Challenges from

Japan'sTrue Internationalization » in DoNAHUE R. (ed) Exploring Japaneseness - on

Japanese enactments of culture and conscoiusness, Westport, Ablex Publishing, p. 197-222.

SUZUKI T. 1975.Tozasareta gengo - Nihongo no sekai. Tokyo, Shinchô Sensho.

WATANABE, S. 1974. Nihongo no kokoro. Tokyo, Kôdansha Gendai shinsho.

WATsujI, T. 1962. "Mono no aware" nitsuite, in Watsuji Tetsurô Zenshû. Tokyo, Inawammi Shoten.

ҮАмамото, Н. 2005. " Rapport japonais “les minorités en Droit public interne" », in Association Henri Capitant des amis de la culture juridique française, Les Minorités. Journées mexicaines LII/ 2002. Mexico, Instituto de investicaciones jurídicas : 759-770.

\section{NOTES}

1. Au Japon sont aussi exclus de cette comptabilité les membres des forces américaines (près de 50000 hommes ; source : http://www.usfj.mil/) et de leurs familles.

2. Données tirées du Human Development Report (consacré aux migrations) publié par le Programme des Nations unies pour le développement (PNUD) (2009: 143-148).

3. Données tirées du PNUD, op.cit. : 155-158.

4. Par exemple, la scolarisation des enfants étrangers n'est pas obligatoire au Japon.

5. Cité d'après ibid. : 38 
6. Données de l'INSEE.

7. Avant même la promulgation de la loi, par une simple circulaire administrative (voir Yamamoto 2005).

8. Malgré un programme d'aide au retour surtout vers la Corée du Nord avec le soutien de la Croix-Rouge.

9. Nozaki et al. (2006) http://www.japanfocus.org/-Yoshiko-Nozaki/2220, consulté le 7 novembre 2010.

10. Je ne décris pas les complications liées à la nécessité pour les Coréens de choisir un rattachement soit à la Corée du Sud, soit à la Corée du Nord.

11. À cette date, ils étaient 700000 tandis que, de manière cumulée depuis 1952, ils étaient par ailleurs 160000 à avoir ré-obtenu la nationalité japonaise.

12. Mais les naturalisations se sont poursuivies pour atteindre en 2003 presque 280000 de manière cumulée depuis 1952.

13. À partir de 1907, quand l'émigration aux États-Unis n'est plus possible, l'émigration japonaise se tourne massivement vers le Brésil. Il y a là-bas plus d'un million de descendants de Japonais. Voir sur cette question et la situation des Brésiliens au Japon, la thèse de P. Cherrier, Entre Japon et Brésil : identités décalées, Lyon, Université de Lyon-II, 2010. La première annonce de recrutement de Brésiliens descendants de Japonais paraît dans un journal brésilien en 1985 (Sekiguchi, 2002). Leur nombre croît rapidement, il dépasse la centaine en 1987, le millier en 1990.

14. Pour les désigner, on emploie aussi à la place de Citizen, le terme denizen que l'on doit à T. Hammar, Democracy and the Nation State. Aliens, Denizens and Citizens in a World of International Migration, Aldershot, Avebury, 1990.

15. La loi de 1986 ne concernait principalement que les services.

16. 300000 yens pour le chef de famille et 200000 yens pour ses dépendants.

17. Soit 680518 personnes, dix fois plus qu'en France où se trouvent environ 60000 Chinois (INSEE enquête 2004-5). La population étrangère totale au Japon était en 2009 de 2186121 personnes.

18. Dans une autre perspective sur cette caractéristique japonaise voir l'ouvrage suivant: A. L'Hénoret, Le clou qui dépasse, Paris, La Découverte, 1997.

19. Ce sont les îles de Hokkaido et celles plus au nord, dont certaines sont passées sous contrôle russe aujourd'hui et s'étendent jusqu'à la presqu'île de Kamchaka. Les îles Ryukyu ont longtemps payé tribut à la fois à la Chine et au Japon. Elles sont devenues Okinawa, qui n'a été rattachée au Japon qu'en 1879, puis est passée sous administration nord-américaine de 1945 à 1972. Les principales bases militaires américaines s'y trouvent implantées et posent quelques problèmes.

20. Transformation politique engagée sous l'impact de l'imposition de relations commerciales (inégales) par les Occidentaux (après le passage des navires du commodore Perry en 1853).

21. Les années sont comptées d'après les noms pris par chaque empereur au moment de leur accession au pouvoir impérial. L'ère Meiji court donc de 1868 à 1912, l'ère Taisho de 1912 à 1926, l'ère Showa de 1926 à 1989, l'ère Heisei a commencé en 1989.

22. Philippe Pelletier 2007a: 391.

23. Citation de Masuda Yoshio, Junsui bunka no jôken: Nihon bunka wa shôgeki ni dô taeta ka,Tokyo, Kodansha, d'après Philippe Pelletier (2007a: 389).

24. Propos rapporté par Philippe Pelletier, ibid.

25. Motoori Norinaga zenshû (Æuvres complètes de Motoori Norinaga, vol. VIII, Tokyo, Chikuma, 1972 : 442-443 ; cité d'après Berque 2000 : 33. Berque écrit qu'il remercie Araki Tôru de lui avoir indiqué la référence.

26. Dale (1986) cite également Morita en 1909 que je n'ai pas su retrouver.

27. Dale (1986 : 207) relie cette position à celle de Morita.

28. Ibid. : 209 et 212 .

Transcontinentales, $8 / 9$ | 2010 
29. Ibid.: 220-221. L'idée de kanjinshugi sera reprise par Kumon qui la traduit par "contextualisme». Odin discute le terme de Kumon et plus largement la relation avec le pragmatisme philosophique américain et le bouddhisme zen japonais.

30. Dale $1986: 218$.

31. Mais aussi les burakumin, les malades de la lèpre ou de la maladie de Hansen, les victimes de Minamata, etc.

32. Dale $1986: 221$.

33. Intellectuels parmi lesquels Kôtoku Shûsui, dont Chritine Levy a traduit l'ouvrage clé L'impérialisme, le spectre du XX $x^{e}$ siècle,Paris, CNRS Éditions, 2008, en l'assortissant d'une analyse.

34. D'après Ogino (2004: 42) qui cite Kafû (1919) Hanabi.

35. Ogino $2004: 43$.

36. Voir par exemple Yamamoto $2005: 764$.

\section{RÉSUMÉS}

Le Japon a longtemps pratiqué des politiques explicites ou implicites de pureté ethnique, entraînant des discriminations internes à l'égard de populations allogènes ou impures (Aïnous, burakumin, okinawaiens, par exemple) et de comportements de même type à l'égard de populations étrangères provenant d'un nombre restreint de pays en raison de vicissitudes historiques (Corée, Brésil). Aujourd'hui les conditions socio-économiques d'un pays fortement vieillissant et à population décroissante face à la montée en puissance d'un voisin dix fois plus peuplé, apportent cependant une certaine pression de nouvelles immigrations (Philippines, Chine). La réticence historique japonaise semble pourtant perdurer par des pratiques, tant discriminatoires face à des immigrations "politiques" (du Moyen-Orient par exemple) qu'économiques en favorisant notamment le développement de robots d'aide aux personnes âgées ou handicapées plutôt que des aides familiales étrangères. La main-d'œuvre " purement » japonaise accepte et prend des emplois manuels et de service qu'en d'autres pays il faut confier à des populations immigrées; le Japon fait fabriquer à l'extérieur ses vêtements, etc., plutôt que de le faire dans des usines accueillant des travailleurs étrangers et cependant il n'a pas, au contraire, une balance commerciale manufacturière déficitaire. La limitation de l'immigration est donc bien réelle, elle est accompagnée d'un dépassement des arguments économiques employés ailleurs, par des solutions qui exigent des comportements spécifiques de la population. En revanche il semble ne pas y avoir de freins effectifs à l'afflux d'étudiants chinois qui forment de loin la première population étudiante étrangère au Japon. Ici comme en d'autres domaines au regard des différents critères, le Japon parait comme un cas « à part » des pays à haut revenu.

For a long time, Japan has practiced written and unwritten ethnic purity policies. This has led to discrimination against non-native or impure populations (like the Ainu, Burakumin and Okinawans) and similar behaviour towards foreign nationals from certain countries (such as Korea or Brazil) as a result of past events. Today, however, the country is aging, the population is shrinking, and its extremely populous neighbour is growing in power. These changing socioeconomic conditions have led to more pressure for new immigration (from the Philippines and China). Nevertheless, Japanese reticence has not disappeared, and can be seen in discriminatory practices targeting "political" immigration (from the Middle East, for example) and economic practices (like encouraging the development of robots to help elderly or handicapped people 
instead of hiring foreign help). "Pure" Japanese workers accept manual and service jobs, and do not leave them to immigrants as often happens in other countries. Japan produces clothing and other products overseas rather than setting up factories that would employ foreign workers. And yet the country has a positive balance of trade in manufacturing. Limits on immigration are very real, and are accompanied by solutions requiring the population to act in certain ways, bypassing the economic arguments used elsewhere. However, it appears that there are no real limits to the inflow of Chinese students, who represent the largest foreign student group in Japan. In this domain and others, different factors would seem to indicate that Japan is once again an "exception" amongst wealthy countries.

INDEX

Thèmes : migration, mobilité

Index géographique : Japon

\section{AUTEUR}

\section{MARC HUMBERT}

Professeur d'économie politique à l'université de Rennes 1,

Directeur de la Maison franco-japonaise de Tokyo (mfj-Umifre 19), http://

www.mfj.gr.jp/

direction@mfj.gr.jp 